\title{
Exponential Functions and Laplace Transforms for Alpha Derivatives
}

\author{
ELVAN AKIN-BOHNER and MARTIN BOHNER \\ Department of Mathematics, Florida Institute of Technology \\ Melbourne, Florida 32901 \\ E-mail: eakin@math.unl.edu and bohner@umr.edu
}

\begin{abstract}
We introduce the exponential function for alpha derivatives on generalized time scales. We also define the Laplace transform that helps to solve higher order linear alpha dynamic equations on generalized time scales. If $\alpha=\sigma$, the Hilger forward jump operator, then our theory contains the theory of delta dynamic equations on time scales as a special case. If $\alpha=\rho$, the Hilger backward jump operator, then our theory contains the theory of nabla dynamic equations on time scales as a special case. Hence differential equations, difference equations (using the forward or backward difference operator), or $q$-difference equations (using the forward or backward $q$-difference operator) can be accommodated within our theory. We also present various properties of the Laplace transform and offer some examples.
\end{abstract}

Keywords Alpha derivative, generalized time scale, exponential function, Laplace transform

AMS Subject Classification 39A12, 39A13

\section{INTRODUCTION}

We consider generalized time scales $(\mathbb{T}, \alpha)$ as introduced in [1], i.e., $\mathbb{T} \subset \mathbb{R}$ is a nonempty set such that every Cauchy sequence in $\mathbb{T}$ converges to a point in $\mathbb{T}$ (with the possible exception of Cauchy sequences that converge to a finite infimum or supremum of $\mathbb{T}$ ), and $\alpha$ is a function that maps $\mathbb{T}$ into $\mathbb{T}$. A 
function $f: \mathbb{T} \rightarrow \mathbb{R}$ is called alpha differentiable at a point $t \in \mathbb{T}$ if there exists a number $f_{\alpha}(t)$, the so-called alpha derivative of $f$ at $t$, with the property that for every $\varepsilon>0$ there exists a neighborhood $U$ of $t$ such that

$$
\left|f(\alpha(t))-f(s)-f_{\alpha}(t)(\alpha(t)-s)\right| \leq \varepsilon|\alpha(t)-s|
$$

is true for all $s \in U$. If $\mathbb{T}$ is closed and $\alpha=\sigma$, the Hilger forward jump operator, then $f_{\alpha}=f^{\Delta}$ is the usual delta derivative (see $[4,6,7]$ ), which contains as special cases derivatives $f^{\prime}$ (if $\mathbb{T}=\mathbb{R}$ ) and differences $\Delta f$ (if $\mathbb{T}=\mathbb{Z}$ ). If $\mathbb{T}$ is closed and $\alpha=\rho$, the Hilger backward jump operator, then $f_{\alpha}=f^{\nabla}$ is the nabla derivative (see [3] and [4, Section 8.4]).

In this paper we consider linear alpha dynamic equations of the form

$$
y_{\alpha}=p(t) y \quad \text { with } \quad 1+p(t) \mu_{\alpha}(t) \neq 0,
$$

where $\mu_{\alpha}(t)=\alpha(t)-t$ is the generalized graininess. If the initial value problem

$$
y_{\alpha}=p(t) y, \quad y\left(t_{0}\right)=1
$$

has a unique solution, we denote it by $e_{p}\left(t, t_{0}\right)$ and call it the generalized exponential function. Note that $e_{p}$ also depends on $\alpha$, but we choose not to indicate this dependence as it should be clear from the context. The exponential function satisfies some properties, which are presented in the next section of this paper. Similarly as in [5], the exponential function may be used to define a generalized Laplace transform, which is helpful when solving higher order linear alpha dynamic equations with constant coefficients. We illustrate this technique with an example in the last section. This example features an $\alpha$ which neither satisfies $\alpha(t) \geq t$ for all $t \in \mathbb{T}$ nor $\alpha(t) \leq t$ for all $t \in \mathbb{T}$, and hence this example can not be accommodated in the existing literature on delta and nabla dynamic equations. 


\section{Alpha Derivatives, Exponentials, and Laplace Transforms}

For a function $f: \mathbb{T} \rightarrow \mathbb{R}$ we denote by $f_{\alpha}$ the alpha derivative as defined in the introductory section, and we also put $f^{\alpha}=f \circ \alpha$. Then the following rules (see [4, Section 8.3]) are valid:

- $f^{\alpha}=f+\mu_{\alpha} f_{\alpha}$;

- $(f g)_{\alpha}=f g_{\alpha}+f_{\alpha} g^{\alpha}$ ("Product Rule");

- $\left(\frac{f}{g}\right)_{\alpha}=\frac{f_{\alpha} g-f g_{\alpha}}{g g^{\alpha}}$ ("Quotient Rule").

We may use these rules to find

$$
e_{p}\left(\alpha(t), t_{0}\right)=e_{p}^{\alpha}\left(t, t_{0}\right)=e_{p}\left(t, t_{0}\right)+\mu_{\alpha}(t) p(t) e_{p}\left(t, t_{0}\right),
$$

i.e.,

- $e_{p}\left(\alpha(t), t_{0}\right)=\left[1+p(t) \mu_{\alpha}(t)\right] e_{p}\left(t, t_{0}\right)$,

by putting $y(t)=e_{p}\left(t, t_{0}\right) e_{q}\left(t, t_{0}\right)$,

$$
\begin{aligned}
y_{\alpha}(t) & =e_{p}\left(t, t_{0}\right) q(t) e_{q}\left(t, t_{0}\right)+p(t) e_{p}\left(t, t_{0}\right) e_{q}\left(\alpha(t), t_{0}\right) \\
& =\left[p(t)+q(t)+\mu_{\alpha}(t) p(t) q(t)\right] y(t),
\end{aligned}
$$

i.e.,

- $e_{p} e_{q}=e_{p \oplus q}, \quad$ where $\quad p \oplus q:=p+q+\mu_{\alpha} p q$,

and by putting $y=e_{p}\left(t, t_{0}\right) / e_{q}\left(t, t_{0}\right)$,

$$
\begin{aligned}
y_{\alpha}(t) & =\frac{p(t) e_{p}\left(t, t_{0}\right) e_{q}\left(t, t_{0}\right)-e_{p}\left(t, t_{0}\right) q(t) e_{q}\left(t, t_{0}\right)}{e_{q}\left(t, t_{0}\right) e_{q}\left(\alpha(t), t_{0}\right)} \\
& =\frac{p(t)-q(t)}{1+\mu_{\alpha}(t) q(t)} y(t),
\end{aligned}
$$

i.e.,

$$
\text { - } \frac{e_{p}}{e_{q}}=e_{p \ominus q}, \quad \text { where } \quad p \ominus q:=\frac{p-q}{1+\mu_{\alpha} q} .
$$

Note also that $\ominus q:=0 \ominus q=-q /\left(1+\mu_{\alpha} q\right)$ satisfies $q \oplus(\ominus q)=0$ and that $p \ominus q=p \oplus(\ominus q)$. Again, $\oplus$ and $\ominus$ depend on $\alpha$, but in order to avoid many subscripts we choose not to indicate this dependence as it should be clear from the context. We also remark that the set of alpha regressive functions

$$
\mathcal{R}_{\alpha}=\left\{p: \mathbb{T} \rightarrow \mathbb{R} \mid 1+p(t) \mu_{\alpha}(t) \neq 0 \text { for all } t \in \mathbb{T}\right\}
$$


is an Abelian group under the addition $\oplus$, and $\ominus p$ is the additive inverse of $p \in \mathcal{R}_{\alpha}$.

Now, similarly as in [5], the Laplace transform for functions $x: \mathbb{T} \rightarrow \mathbb{R}$ (from now on we assume that $\mathbb{T}$ is unbounded above and contains 0 ) may be introduced as

$$
\mathcal{L}\{x\}(z)=\int_{0}^{\infty} x(t) e_{\ominus z}^{\alpha}(t, 0) d_{\alpha} t \quad \text { with } \quad z \in \mathcal{R}_{\alpha} \cap \mathbb{R}
$$

whenever this Cauchy alpha integral is well defined. As an example, we calculate $\mathcal{L}\left\{e_{c}(\cdot, 0)\right\}$, where $c \in \mathcal{R}_{\alpha}$ is a constant such that $\lim _{t \rightarrow \infty} e_{c \ominus z}(t, 0)=0$.

Then

$$
\begin{aligned}
\mathcal{L}\left\{e_{c}(\cdot, 0)\right\}(z) & =\int_{0}^{\infty} e_{c}(t, 0) e_{\ominus z}^{\alpha}(t, 0) d_{\alpha} t \\
& =\int_{0}^{\infty}\left[1+\mu_{\alpha}(t)(\ominus z)(t)\right] e_{c}(t, 0) e_{\ominus z}(t, 0) d_{\alpha} t \\
& =\int_{0}^{\infty}\left[1-\frac{\mu_{\alpha}(t) z}{1+\mu_{\alpha}(t) z}\right] e_{c \ominus z}(t, 0) d_{\alpha} t \\
& =\int_{0}^{\infty} \frac{1}{1+\mu_{\alpha}(t) z} e_{c \ominus z}(t, 0) d_{\alpha} t \\
& =\frac{1}{c-z} \int_{0}^{\infty}(c \ominus z)(t) e_{c \ominus z}(t, 0) d_{\alpha} t \\
& =\frac{1}{c-z} \int_{0}^{\infty}\left(e_{c \ominus z}(\cdot, 0)\right)_{\alpha} d_{\alpha} t \\
& =\frac{1}{z-c} .
\end{aligned}
$$

Under appropriate assumptions we can also show

- $\mathcal{L}\left\{x_{\alpha}\right\}(z)=z \mathcal{L}\{x\}(z)-x(0)$

- $\mathcal{L}\left\{x_{\alpha \alpha}\right\}(z)=z^{2} \mathcal{L}\{x\}(z)-z x(0)-x_{\alpha}(0)$;

- $\mathcal{L}\{X\}(z)=\frac{1}{z} \mathcal{L}\{x\}(z), \quad$ where $\quad X(t)=\int_{0}^{t} x(\tau) d_{\alpha} \tau$.

Further results can be derived as in [4, Section 3.10]. 


\section{An ExAmple}

To illustrate the use of our Laplace transform, we consider the initial value problem

$$
y_{\alpha \alpha}-5 y_{\alpha}+6 y=0, \quad y(0)=1, \quad y_{\alpha}(0)=5 .
$$

By formally taking Laplace transforms, we find

$$
\begin{aligned}
0 & =z^{2} \mathcal{L}\{y\}(z)-z y(0)-y_{\alpha}(0)-5[z \mathcal{L}\{y\}(z)-y(0)]+6 \mathcal{L}\{y\}(z) \\
& =\left(z^{2}-5 z+6\right) \mathcal{L}\{y\}(z)-z \\
& =(z-2)(z-3) \mathcal{L}\{y\}(z)-z
\end{aligned}
$$

so that

$$
\mathcal{L}\{y\}(z)=\frac{z}{(z-2)(z-3)}=\frac{3}{z-3}-\frac{2}{z-2}=\mathcal{L}\left\{3 e_{3}(\cdot, 0)-2 e_{2}(\cdot, 0)\right\}(z) .
$$

Hence, if $e_{2}(\cdot, 0)$ and $e_{3}(\cdot, 0)$ exist, we let

$$
y=3 e_{3}(\cdot, 0)-2 e_{2}(\cdot, 0),
$$

and then

$$
y_{\alpha}=9 e_{3}(\cdot, 0)-4 e_{2}(\cdot, 0) \quad \text { and } \quad y_{\alpha \alpha}=27 e_{3}(\cdot, 0)-8 e_{2}(\cdot, 0)
$$

so that indeed $y(0)=3-2=1, y_{\alpha}(0)=9-4=5$, and $y_{\alpha \alpha}-5 y_{\alpha}+6 y=0$.

Let us now consider several special cases of this example.

(a) $\mathbb{T}=\mathbb{R}$ and $\alpha(t)=t$ for all $t \in \mathbb{T}$. Then $e_{c}(t, 0)=e^{c t}$ for any constant $c \in \mathbb{R}$, and the solution is given by

$$
y(t)=3 e^{3 t}-2 e^{2 t}
$$

(b) $\mathbb{T}=\mathbb{N}_{0}$ and $\alpha(t)=2 t+1$ for all $t \in \mathbb{T}$. Note that $e_{c}(\cdot, 0)$ is only defined on

$$
\left\{t_{m}=2^{m}-1 \mid m \in \mathbb{N}_{0}\right\} \subset \mathbb{T} .
$$

Since $\mu_{\alpha}(t)=t+1$, we find that $e_{c}$ satisfies

$$
e_{c}\left(t_{k+1}, 0\right)=\left(1+c \mu_{\alpha}\left(t_{k}\right)\right) e_{c}\left(t_{k}, 0\right)=\left(1+c 2^{k}\right) e_{c}\left(t_{k}, 0\right),
$$


TABLE 1. $y=3 e_{3}(\cdot, 0)-2 e_{2}(\cdot, 0)$ for $(\mathrm{b})$

\begin{tabular}{c|ccccc}
$t$ & $e_{2}(t, 0)$ & $e_{3}(t, 0)$ & $y(t)$ & $y_{\alpha}(t)$ & $y_{\alpha \alpha}(t)$ \\
\hline 0 & 1 & 1 & 1 & 5 & 19 \\
1 & 3 & 4 & 6 & 24 & 84 \\
3 & 15 & 28 & 54 & 192 & 636 \\
7 & 135 & 364 & 822 & 2736 & 8748 \\
15 & 2295 & 9100 & 22710 & 72720 & \\
31 & 75735 & 445900 & 1186230 & &
\end{tabular}

and hence we obtain for constant $c \in \mathcal{R}_{\alpha}$

$$
e_{c}\left(t_{m}, 0\right)=\prod_{k=0}^{m-1}\left(1+c 2^{k}\right) .
$$

See Table 1 for some numeric values. Note that $y_{\alpha \alpha}-5 y_{\alpha}+6 y=0$ in each row.

(c) $\mathbb{T}=\mathbb{Z}$ and $\alpha(t)=t-2$ for all $t \in \mathbb{T}$. Note that $e_{c}(t, 0)$ is only defined for all even integers. Since $\mu_{\alpha}(t) \equiv-2$, we find that $e_{c}$ satisfies

$$
e_{c}(\alpha(t), 0)=\left(1+c \mu_{\alpha}(t)\right) e_{c}(t, 0)=(1-2 c) e_{c}(t, 0)
$$

and hence we obtain for constant $c \neq 1 / 2$

$$
e_{c}(t, 0)=(1-2 c)^{-t / 2} \text {. }
$$

See Table 2 for some numeric values.

(d) $\mathbb{T}=\mathbb{Z}$ and $\alpha(t)=t+1+2(-1)^{t}$ for all $t \in \mathbb{T}$. Note that while in the previous two examples $\alpha(t) \geq t$ for all $t \in \mathbb{T}$ and $\alpha(t) \leq t$ for all $t \in \mathbb{T}$, respectively, none of these properties hold in the current example. This time $\alpha: \mathbb{T} \rightarrow \mathbb{T}$ is additionally a bijection and hence $e_{c}(t, 0)$ is defined on the entire set $\mathbb{T}$ when $c \notin\{-1 / 3,1\}$. We have

$$
\mu_{\alpha}(t)=1+2(-1)^{t}= \begin{cases}3 & \text { if } t \text { is even } \\ -1 & \text { if } t \text { is odd }\end{cases}
$$


TABLE 2. $y=3 e_{3}(\cdot, 0)-2 e_{2}(\cdot, 0)$ for $(\mathrm{c})$

\begin{tabular}{c|ccccc}
$t$ & $e_{2}(t, 0)$ & $e_{3}(t, 0)$ & $y(t)$ & $y_{\alpha}(t)$ & $y_{\alpha \alpha}(t)$ \\
\hline-6 & -27 & -125 & -321 & & \\
-4 & 9 & 25 & 57 & 189 & \\
-2 & -3 & -5 & -9 & -33 & -111 \\
0 & 1 & 1 & 1 & 5 & 19 \\
2 & $-0 . \overline{3}$ & -0.2 & $0.0 \overline{6}$ & $-0.4 \overline{6}$ & $-2.7 \overline{3}$ \\
4 & $0 . \overline{1}$ & 0.04 & $-0.10 \overline{2}$ & $-0.08 \overline{4}$ & $0.19 \overline{1}$
\end{tabular}

As an example we calculate $e_{2}(t, 0)$ for some values of $t$. Since $e_{2}(0,0)=1$ and $\alpha(0)=3$, we find

$$
e_{2}(3,0)=e_{2}(\alpha(0), 0)=\left(1+2 \mu_{\alpha}(0)\right) e_{2}(0,0)=7 \text {. }
$$

Next,

$$
e_{2}(2,0)=e_{2}(\alpha(3), 0)=\left(1+2 \mu_{\alpha}(3)\right) e_{2}(3,0)=-7,
$$

and similarly,

$$
e_{2}(5,0)=-7^{2}, \quad e_{2}(4,0)=7^{2}, \quad e_{2}(7,0)=7^{3}, \quad e_{2}(6,0)=-7^{3}
$$

and so on. In general, we find

$$
e_{c}(t, 0)= \begin{cases}\{(1-c)(1+3 c)\}^{t / 2} & \text { if } t \text { is even } \\ (1-c)^{(t-3) / 2}(1+3 c)^{(t-1) / 2} & \text { if } t \text { is odd }\end{cases}
$$

which can be written in closed formula as

$$
e_{c}(t, 0)=\frac{\{(1-c)(1+3 c)\}^{\lfloor t / 2\rfloor}}{(1-c)^{\chi(t)}}=\frac{\{(1-c)(1+3 c)\}^{\lfloor t / 2\rfloor}}{(1-c)^{\lceil t / 2-\lfloor t / 2\rfloor\rceil}},
$$

where $\chi=\chi_{2 \mathbb{Z}+1}$ is the characteristic function for the odd integers. Again we refer to Table 3 for some numeric values. 
TABle 3. $y=3 e_{3}(\cdot, 0)-2 e_{2}(\cdot, 0)$ for $(\mathrm{d})$

\begin{tabular}{c|ccccc}
$t$ & $e_{2}(t, 0)$ & $e_{3}(t, 0)$ & $y(t)$ & $y_{\alpha}(t)$ & $y_{\alpha \alpha}(t)$ \\
\hline 0 & 1 & 1 & 1 & 5 & 19 \\
1 & -1 & -0.5 & 0.5 & -0.5 & -5.5 \\
2 & -7 & -20 & -46 & -152 & -484 \\
3 & 7 & 10 & 16 & 62 & 214 \\
4 & 49 & 400 & 1102 & 3404 & 10408 \\
5 & -49 & -200 & -502 & -1604 & -5008 \\
6 & -343 & -8000 & -23314 & -70628 & \\
7 & 343 & 4000 & 11314 & 34628 & \\
8 & 2401 & 160000 & 475198 & & \\
9 & -2401 & -80000 & -235198 & &
\end{tabular}

\section{REFERENCES}

[1] C. D. Ahlbrandt, M. Bohner, and J. Ridenhour. Hamiltonian systems on time scales. J. Math. Anal. Appl., 250:561-578, 2000.

[2] E. Akın, L. Erbe, B. Kaymakçalan, and A. Peterson. Oscillation results for a dynamic equation on a time scale. J. Differ. Equations Appl., 7:793-810, 2001.

[3] F. M. Atıcı and G. Sh. Guseinov. On Green's functions and positive solutions for boundary value problems on time scales. J. Comput. Appl. Math., 2002. Special Issue on "Dynamic Equations on Time Scales", edited by R. P. Agarwal, M. Bohner, and D. O'Regan. To appear.

[4] M. Bohner and A. Peterson. Dynamic Equations on Time Scales: An Introduction with Applications. Birkhäuser, Boston, 2001.

[5] M. Bohner and A. Peterson. Laplace transform and Z-transform: Unification and extension. Methods Appl. Anal., 2002. To appear.

[6] S. Hilger. Analysis on measure chains - a unified approach to continuous and discrete calculus. Results Math., 18:18-56, 1990.

[7] B. Kaymakçalan, V. Lakshmikantham, and S. Sivasundaram. Dynamic Systems on Measure Chains, volume 370 of Mathematics and its Applications. Kluwer Academic Publishers, Dordrecht, 1996.

[8] W. G. Kelley and A. C. Peterson. Difference Equations: An Introduction with Applications. Academic Press, San Diego, second edition, 2001. 